\title{
ON THE EVOLUTION OF THE CENTRAL STARS
}

\author{
Willi Deinzer \\ (University of Heidelberg, Germany)
}

A most puzzling feature of the evolution of the central stars of planetary nebulae is their rapid contraction (O'Dell, 1963; Seaton, 1966); assuming homologous contraction, an enormous amount of gravitational energy is released therefore. For reasons of hydrostatic equilibrium a large part of this energy must be radiated away. As this does not show up in the luminosity of the central stars it must either leave them by some other means - e.g. neutrinos (Vila, 1966) - or it does not originate from the outset; in other words, the actual contraction must deviate strongly from the homologous way. I should like to discuss briefly the second possibility.

For this purpose the following type of stellar model is considered: The central stars should consist of a core, which contains almost all of the stellar mass and the radius of which has almost decreased to the limit set by complete degeneracy (Schwarzschild, $1958, \S 26)$. They should further consist of an envelope containing a negligible amount of mass but of very extended radius. If at the end of the mass-loss phase producing the planetary nebula such a star has no nuclear-energy sources available anymore, it will start contracting. This contraction will now proceed in a strongly non-homologous way. The envelope will change its radius by a large amount, which is accompanied by a negligible amount of gravitational-energy release because of the negligible amount of mass taking part in this contraction. The core can change its radius only very little; but in view of the large amount of mass taking part in this contraction an appreciable amount of gravitational energy could be released. Besides, thermal energy could be released due to the effect that contraction of a degenerate configuration will lead to cooling.

Is it possible in this way to obtain evolutionary time-scales and evolutionary tracks comparable to those observed for the central stars? To answer this question, calculations were carried out for a simplified model. Its partially degenerate core is assumed to be isothermal (Schwarzschild, 1958, §13); the prevailing opacity of its radiative envelope should be due to Thomson scattering only. The solutions for the envelope can be obtained analytically and hence can easily be fitted to the core solutions. Results obtained are shown in Figure 1 . They belong to stars of $0.4 M_{\odot}$ consisting of a helium core and of a population-II envelope. The tracks differ in the mass content of the envelope $\left(10^{-2}, 10^{-3}, 10^{-4}\right.$ of the total mass). They are limited towards high luminosities by the restriction that radiation pressure in the envelope can at the most equal the total pressure; and they are limited towards the left by the white-dwarf

Osterbrock and O'Dell (eds.), Planetary Nebulae, 428 430. ․ I.A.U. 


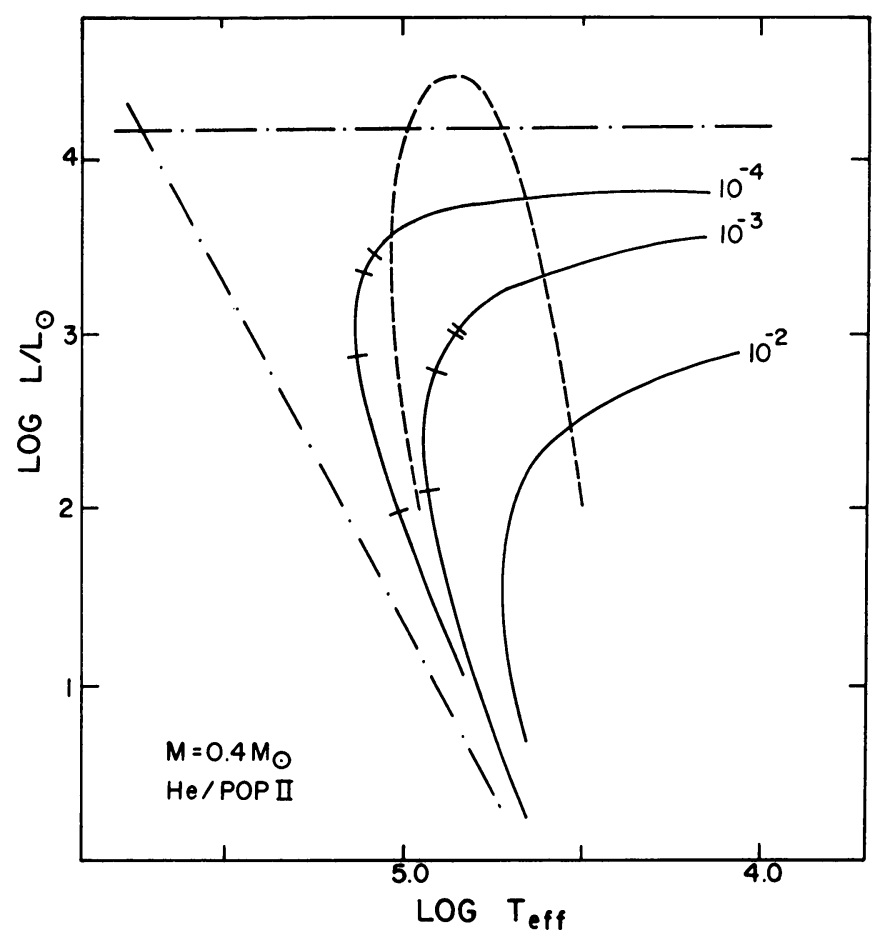

FIG. 1. Evolutionary paths for theoretical stars whose atmospheres contain the designated fraction of the total mass of the star. The dashed line indicates the Harman-Seaton sequence: the upper dash-dot line designates the radiation-pressure boundary, while that on the left is the complete degeneracy boundary. The lower three bars on the evolutionary paths indicate the stellar positions at times of $10^{2}$, $10^{3}$ and $10^{4}$ years.

radius corresponding to $0.4 M_{\odot}$. Along each track the energy release from the core was calculated; together with the luminosity a time-scale was obtained. Bars on the tracks indicate how far a star has evolved after $10^{2}, 10^{3}$ and $10^{4}$ years; a star evolves indeed from high luminosities and large radii to the white-dwarf region. Similar results were obtained for a star of $0.5 M_{\odot}$ consisting of a carbon core and a helium envelope.

Finally let me summarize the simplifications of the present models. There is first the assumption of an isothermal core. It could be justified, if the nebula is produced in a shell-source burning stage of stellar evolution; for this reason also the chemical discontinuity was introduced. Since, in the degenerate state of matter, pressure and density are very insensitive to the temperature, the hydrostatics does not depend very strongly on the assumed temperature distribution (Hayashi et al., 1962, §6E.1). There is next the assumption that all the energy released from the core is transported immediately to the outside of the star. As Baglin (1966) pointed out, the transport timescales are of the order of $10^{6}$ years, even in the case of heat conduction by degenerate 
electrons. Hence, only a small part of the energy from the core will become available during the central-star stage and the evolution of the discussed model stars would proceed faster. Making the envelope a little thicker in mass and taking its gravitationalenergy release into account would probably help to extend the time-scale. Thus stars starting at the top of the Harman-Seaton sequence with a very extended envelope and a highly contracted core could in principle account for the short evolutionary time-scales during which the central stars move through the falling branch of this sequence (for further details see Deinzer, 1967).

\section{References}

Baglin, A. (1966) private communication.

Deinzer, W. (1967) Z. Astrophys., 67, 342.

Hayashi, C., Hoshi, R., Sugimoto, D. (1962) Progress. theor. Phys., Suppl. 22.

O’Dell, C.R. (1963) Astrophys. J., 138, 67.

Schwarzschild, M. (1958) Structure and Evolution of the Stars, Princeton University Press, Princeton.

Seaton, M. J. (1966) Mon. Not. R. astr. Soc., 132, 113.

Vila, S.C. (1966) Astrophys. J., 146, 437. 